\title{
Influence of Phase Change Materials (PCMs) on the thermal performance of building envelopes
}

\author{
Hongxia Zhou, ${ }^{1, *}$, Ake Fransson ${ }^{1}$, and Thomas Olofsson ${ }^{1}$ \\ ${ }^{1}$ Department of Applied Physics and Electronics, Umeå University
}

\begin{abstract}
To understand the influence of PCM wall configurations on the thermal performance of building envelopes, an explicit finite element model of heat transfer from indoor to outdoor (or vice versa) is developed. The accuracy of this model is first validated against the electrical circuit analogy model, and then compared with the experimental data measured in a Hot-Box device. A good agreement between the simulation results and experimental results is obtained. The results of this study show that the PCM configuration layer sequence significantly will affect the thermal performance of building envelopes and that the FEM model developed is a promising tool, which after some more development may be used for optimising PCM wall configurations.
\end{abstract}

\section{Introduction}

To maintain a good (if not optimal) thermal indoor climate, an effective and efficient energy storage and release system is desired. This system is of particular importance in areas of large diurnal variation in outdoor temperature and solar radiation, since it can not only decrease the supplied energy demand for heating and cooling, but also improve the indoor thermal comfort (i.e. small indoor temperature fluctuation).

Therefore, investigations on such solutions have gained more and more attention [1-5] during the last decades. A variety of strategies for enhancing the thermal performance (i.e. energy storage and release) in building component and/or envelopes has been presented.

Conventionally, heavy materials such as, soil [6], concrete [7], brick, stones [8], can be used to build thick walls, so that the envelopes can supply sufficient thermal storage capacity, and moderate the indoor temperature oscillations and further improve the level of thermal comfort. This strategy has turned out to be quite effective, but it is contrary to the design trend [5] of modern buildings using glass walls as well as structures with lower mass and thermal storage capacity.

As opposed to the conventional strategies mentioned above, a novel strategy has been developed, in which phase change materials (PCMs) are integrated with the building envelopes. Note that PCMs are substances (e.g. polynary fatty acid [9], paraffin and crystalline hydrate [5]) that undergo a phase transition with a heat absorption/release at a narrow temperature interval. It was reported in [10] that the thermal performance of 5 $\mathrm{cm}$ PCM wallboard was approximately equivalent of using $23 \mathrm{~cm}$ concrete. Also, the results in [11, 12] showed for sufficient conditions, that the peak temperature in rooms with PCMs on the walls can be reduced significantly $\left(\sim 4{ }^{\circ} \mathrm{C}\right.$ during the day) and that about $25-30 \%$ of the cooling demand can be saved. Moreover, PCMs are versatile and flexible and their application is not restricted for only cooling or heating. They work well at different weather conditions during winter and summer [13] and are claimed to meet different engineering purposes as easy implementation and cost-effectiveness [1-5, 14]. Therefore, PCMs have been recognised $[5,15]$ as promising candidates for energy storage and indoor climate control.

To enhance and optimise the thermal performance of PCMs integrated in building envelopes, a variety of numerical and/or experimental studies [10-12, 16] have been carried out. Correspondingly, many numerical and experimental methods have been proposed. For instance, in [16], an implicit finite difference method was used to simulate the thermal performance of concrete walls containing microencapsulated PCMs. The accuracy of their simulation results was validated against experimental data acquired from a small-scale lab testing equipment. Instead of a small-scaled room, Chandra et al. [10], used a full-scale rectangular room with the dimension of $6 \times 5 \times 4 \mathrm{~m}$, where a PCM wall of smaller thickness was used. An effective heat capacity approach was used to assess the control of the PCM wall on the indoor air temperature. Ismail and Castro [11] used a similar full-scale outdoor test-room, but with a smaller dimension of $2.8 \times 2.2 \times 2.0 \mathrm{~m}$. They developed an explicit finite difference model for the simulation of the transient heat transfer process in the walls. A good agreement was obtained between the measured temperatures and the results of the explicit finite element simulations. In [12], a novel electrical circuit analogy

* Corresponding author: hongxia.zhou@umu.se 
method was developed to assess the heat transfer performance of PCM walls. In addition, the location of the PCM layer is important in order to optimize the thermal performance of the building envelope. Jin et al. [17] did experimental studies of how the effect of PCM position influenced the surface temperature and maximum heat flux. They concluded that there is an optimal location of the PCM to give a lower heat flux and longer load shifting time. Gounni and Alami [18] also investigated optimal locations of PCM in building envelopes. They kept the wall thickness constant with and without PCM for comparison and concluded that the optimal location of PCM should be close to the heat source. Zhu et al. [19] studied the performance of double layer PCM in TRNSYS to meet the requirement of PCM in both cooling and heating seasons. The PCM they used was mainly paraffin (80\%). Heim and Wieprzkowicz [20] studied the positioning of the PCM layer making use of ESP-r software. They found that the most appropriate choice would be an exterior position of the PCM when taking into account the current indoor and meteorological conditions.

To summarize, the implementation of these numerical and experimental methods has contributed to an improved understanding on the thermal behaviour of PCMs. Despite that, there are still challenges open to be addressed, when it comes to the choice of the configuration of layer splitting and material sequencing in building envelope constructions (see Fig. 1). The challenges involve, more specifically:

1) Further research on the systematic design of PCM walls: Most of the aforementioned studies [10, 11, 16] focus on analysing the thermal performance of building envelopes integrated with only one layer of PCM material. Only a few studies have been made investigating the geometrical layer splitting and the material sequence in the design of PCM walls, e.g [21],

2) Need for well-established computational tools to support the design: Such a tool would be in high demand for guiding, evaluating, and optimisation of PCM wall configurations. The accuracy, reliability, stability and flexibility of the numerical tools will highly affect the quality and utility of the design output. Thus, such tools should be carefully validated, prior to the use in a design process.

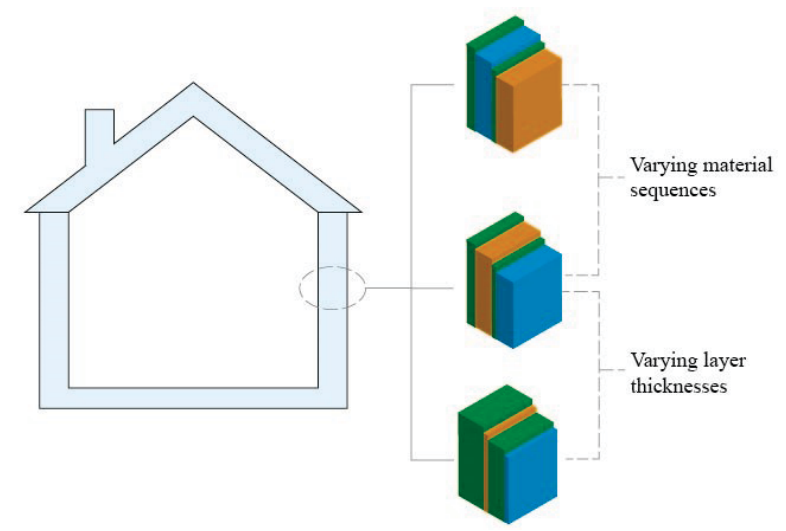

Fig. 1. Schematic of varying wall configuration (Note that the colours refer to different materials, e.g. concrete as blue, bricks as green, PCMs as orange).
To address the research challenges listed above, both numerical and experimental studies are carried out in this study. The aim of this study is to contribute with 1) knowledge about the influence of the configuration of layer splitting and material sequencing using PCMs in building envelope constructions, and 2) further develop a Finite Element Method (FEM) for the simulation of heat transfer across walls with PCMs in building envelopes.

The accuracy of this eFEM tool is first validated against the electrical circuit analogy model. Then, simulated data are compared with experimental data derived using a small-scale hot-box with the dimensions $0.3 \times 0.25 \times 0.2 \mathrm{~m}$. More information about the experimental set-up is given in Section 3.

We are contributing with introductory knowledge of how PCMs influence the temperature profiles in multilayer walls. Further development and refinement of the simulation model will, in future studies, give us a tool for how to optimize the effectiveness of short time heat storages in building constructions, value and guide us showing the potential in different design solutions suggested in future lab- and full-scale tests.

The structure of this paper is organised as follows. First, an explicit FEM model of simulating the heat transfer and temperature profiles of PCM walls is presented in Section 2. This section also includes the verification of the FEM model against the electrical circuit analogy model. In Section 3, the experimental comparisons are presented and the influence of PCMs on the thermal performance of building envelopes are discussed. Finally, in Section 4, the main conclusions are drawn, and recommendations for future work are made.

\section{Modelling of PCM thermal performance}

An explicit FEM is used to analyse the heat transfer through a wall, on the basis of the one-dimensional heat transfer equation:

$$
k \frac{\partial^{2} T}{\partial x^{2}}=\rho c_{p} \frac{\partial T}{\partial t}
$$

where $T$ is the temperature, and $k$ is the thermal conductivity coefficient, $\rho$ is the material density, and $c_{p}$ refers to the specific heat capacity.

\subsection{Explicit FEM approach}

Figure 2 shows the general working procedure of the explicit FE-method. The wall is discretised into $M x-1$ elements from the inside to the outside.

During the simulation period of $0 \sim t, N t-1$ time steps are prescribed. The time step size is $0.001 \mathrm{~s}$ and the element size is $1 \mathrm{~mm}$. Depending on the calculation time $t$ and wall thickness $x$, the number of time steps and elements will increase/decrease correspondingly.

For element $l$ at the inside wall boundary, the heat transfer equation is expressed as,

$$
k \frac{T_{2}^{t}-T_{1}^{t}}{\Delta x}+h_{i}\left(T_{i n}^{t}-T_{1}^{t}\right)=\rho c_{p} \frac{\Delta x}{2}\left(\frac{T_{1}^{t+\Delta t}-T_{1}^{t}}{\Delta t}\right)
$$




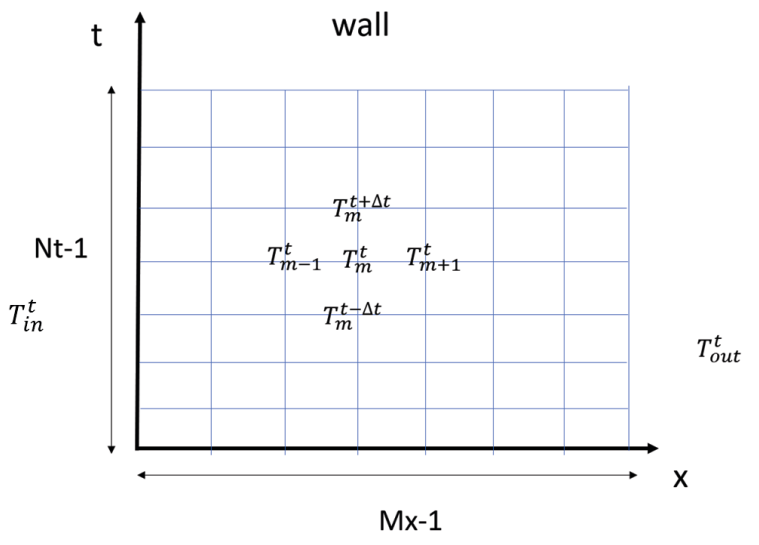

Fig. 2. Working procedure of the explicit FE method.

The inside air temperature of the box $\left(T_{i n}\right)$ is set to follow the experimental values. The heat transfer coefficient at the inside surface $\left(h_{i}\right)$ in the model simulation is thus obtained by numerical calibration with experimental data.

For the element $M x-1$ at the outside boundary, the heat transfer equation is:

$$
k \frac{T_{N-1}^{t}-T_{N}^{t}}{\Delta x}+h_{o}\left(T_{\text {out }}^{t}-T_{N}^{t}\right)=\rho c_{p} \frac{\Delta x}{2}\left(\frac{T_{N}^{t+\Delta t}-T_{N}^{t}}{\Delta t}\right)
$$

The outside air temperature $\left(T_{\text {out }}\right)$ is set to a constant value of $23^{\circ} \mathrm{C}$. The value of heat transfer coefficient on the outside $\left(h_{o}\right)$ is set to be $8 \mathrm{~W} /\left(\mathrm{m}^{2} \mathrm{~K}\right)$ according to [16].

Regarding the in-between elements, the equation is:

$$
k \begin{gathered}
T_{m-1}^{t}-T_{m}^{t} \\
\Delta x
\end{gathered} T_{m+1}^{t}-T_{m}^{t}=\rho c_{p}{ }_{2}^{\Delta x}\left(\begin{array}{c}
T_{m}^{t+\Delta t}-T_{m}^{t} \\
\Delta t
\end{array}\right)
$$

\subsection{PCM material and wall configuration}

The PCM material used in the experiment is Climsel 28, purchased from the Swedish company Climator Sweden AB. A transition at $28^{\circ} \mathrm{C}$ was chosen for the PCM since we do not have access to a cooling equipment. Thus we are limited to the room temperature at around $23^{\circ} \mathrm{C}$ as our lowest operative temperature. Small bags of PCM are packed in a net making a layer as shown in Fig.3.

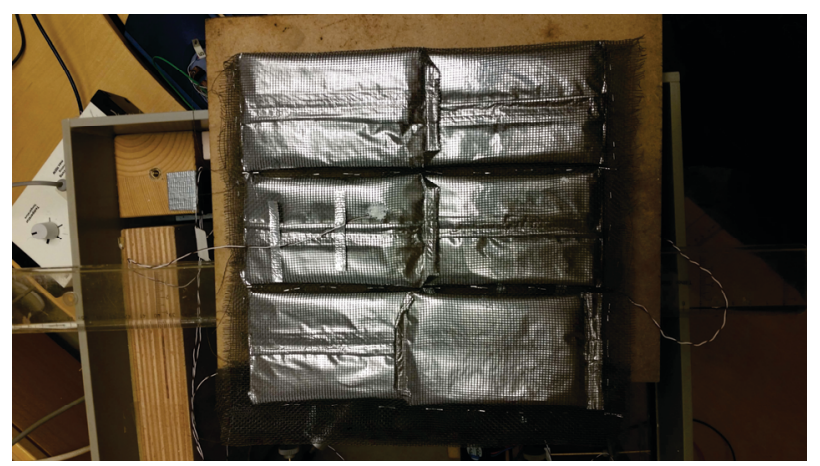

Fig.3. PCM bags packed in a net.

The blue triangles in Fig. 4 are experimental enthalpy data received from the PCM provider. The green circles stand for the specific heat capacity $\left(c_{p}\right)$ derived from equation $d H / d T$. The red curve refers to the specific heat of $c_{p}$, which is derived from the curve fitting of the green circles. The area under the $c_{p^{-}}$-curve reflects the latent heat of the PCM-transition.

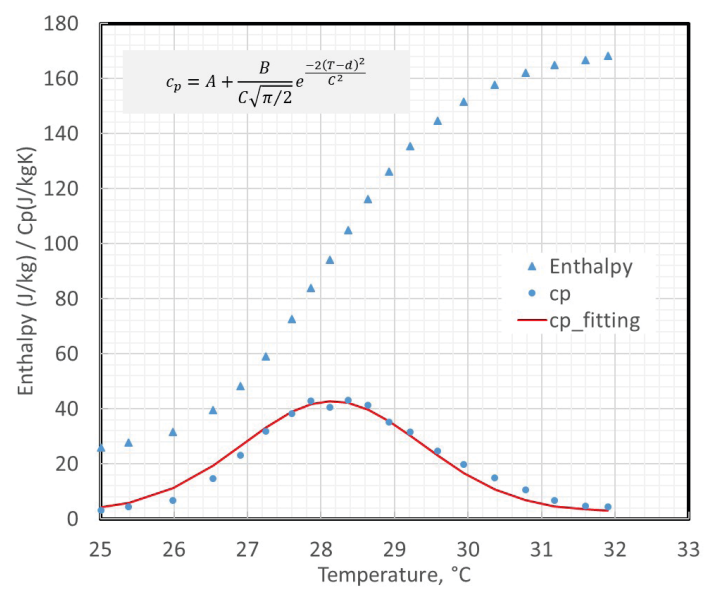

Fig. 4. Enthalpy and specific heat $\left(c_{p}\right)$ of PCM (i.e. Climsel 28) in which $\mathrm{A}=2.70132 ; \mathrm{B}=122.94232 ; \mathrm{C}=2.45197 ; \mathrm{d}=28.15342$.

In this study, there are three kinds of wall configurations (See Fig. 5) to be analysed, namely:

I: A one layer wall with different wall thicknesses $(1 \mathrm{~cm}$ and $2 \mathrm{~cm}$ ). In this configuration, only extruded polystyrene material is considered. Note that, for simplification, the extruded polystyrene is abbreviated as XPS and used hereinafter;

II: A two layer wall (XPS and PCM) with two different material sequences (XPS - PCM and PCM - XPS). The overall thickness and the thicknesses of each layer are the same in both cases;

III: A three layer wall (XPS - PCM - XPS) with varying thicknesses of each layer and varying overall wall thickness.

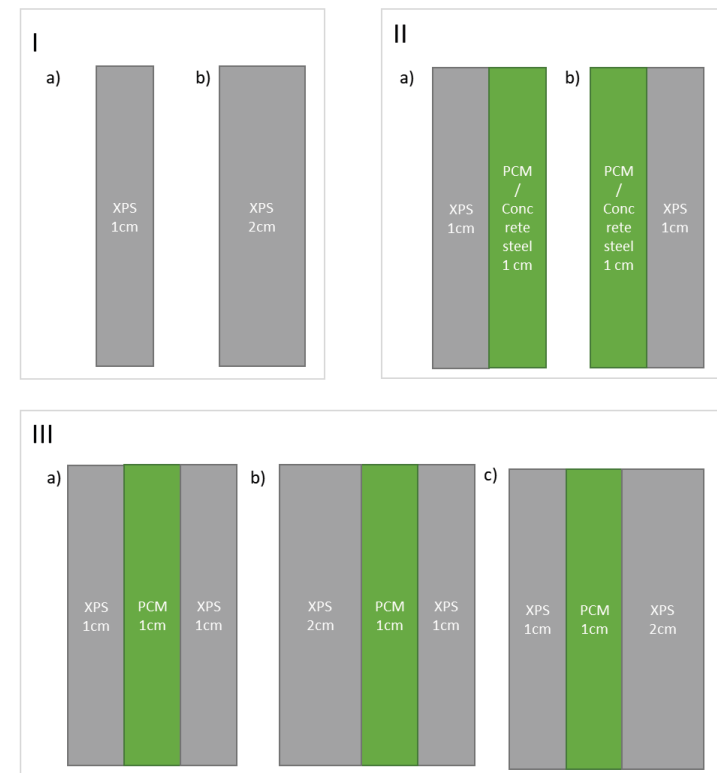

Fig. 5. Wall configurations. Note that the wall's left side is the inside and the right side is the outside. 
Note that the thermal performances of almost all the configurations are discussed in Section 3 of "Model validation", except for the configuration II. Such a wall configuration is analysed in the upcoming section for the purpose of FEM model verification.

\subsection{FEM model verification}

The accuracy of the explicit FEM results is verified by comparison with that obtained from the electrical circuit analogy model introduced by Gori et al. [21].

The working mechanism of the electrical circuit analogy method is to make an analogy of heat flow with the electrical currant. Gori et al. [21] sequenced insulating and thermally conductive layers. The XPS material is here considered as an insulation layer, while concrete/steel is considered as a conduction layer.

More calculation details about the electrical circuit analogy method can be found in [21].

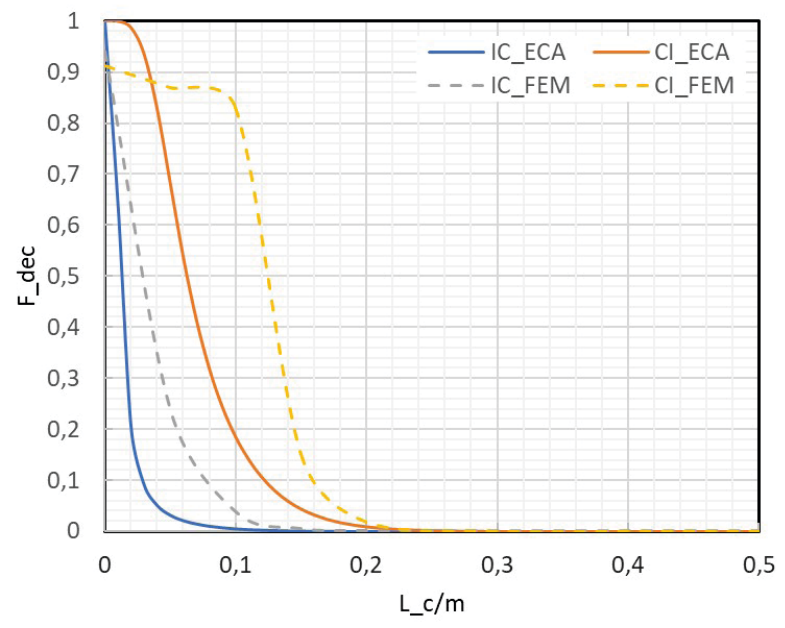

Fig. 6. Comparison of the results of FEM model and electrical circuit analogy (ECA) model based on the data from [21].

Fig. 6 shows that with the thermal performance of the wall configuration II-a and II-b (See Fig. 5), a qualitatively good agreement between the two models is observed. $F_{-}$dec in $\mathrm{Y}$-axis is the temperature decrement factor defined as $F_{-d e c}=T_{I n} / T_{o} . T_{I n}$ and $T_{o}$ are the temperature variation at the inside and outside of the wall. $L \_c$ in $\mathrm{X}$-axis is the thickness of the conduction layer.

Note that, the temperatures in $F \_d e c$ are normalised by using

$$
T_{\text {nor }}=\frac{T-23}{T_{\text {max }}-T_{\text {min }}}
$$

in which, $T_{\max }$ is $40^{\circ} \mathrm{C}$ at inside boundary and $T_{\min }$ is $23^{\circ} \mathrm{C}$ at the outside boundary.

Moreover, it can be observed from Fig. 6 that the results of the electrical circuit analogy model show a small temperature change across the wall. This might be attributed to the relatively small layer thickness used in our study. More in-depth investigations on discrepancies are to be performed in future work.

\section{Model validation against lab tests}

In the validation of the accuracy of the explicit FEM model, presented above, a small-scale experimental setup has been used. Its basic working mechanism and the results of the model validation are discussed in this section.

\subsection{Experimental set-up}

Fig. 7 shows the experimental setup of the small-scale test, where a hot-box has been used, both in the measurements with and without PCM.

The inside temperature of the hot-box is controlled by a heating source, which is connected to a regulator. However in these experiments, the controller is set at its maximum value and the heater will be on all the time. This gives a maximum temperature around $40^{\circ} \mathrm{C}$, obtained when steady-state heat transfer conditions are achieved. The hot-box is located in an office room with an air temperature varying less than $1{ }^{\circ} \mathrm{C}$ around $23^{\circ} \mathrm{C}$. The temperature increase at the inside of the box corresponds to a possible variation in the outside temperature of an assumed building.

One side of hot-box is covered with the PCM material, while the other three sides are covered by glass or wooden board, and used for other purposes. Temperature sensors are attached on both sides of each layer. A small fan is used to increase the convection and equalize the vertical temperature gradient inside of the Hot-Box. The air temperatures at the inside and the outside of the hot-box are measured and collected by using a data logger.

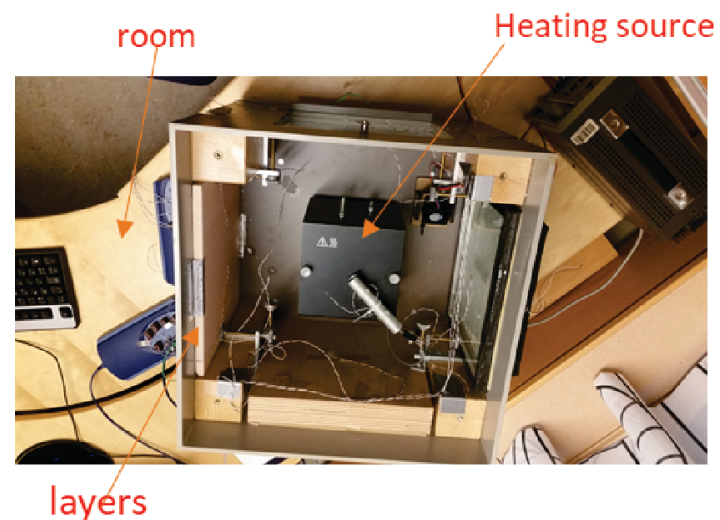

Fig. 7. Experimental set-up of the hot-box. 


\subsection{Results of model validation}

\section{a) One layer (Config. I-a and b)}

Simulated temperature profiles for the wall configuration I-a and I-b (See Fig. 5) are shown in Fig.8. Lines from top to bottom represent temperatures at different wall depths from the inside to the outside. The top line, in Fig. 8. is the air temperature inside of the hotbox and the second line is the temperature on the internal surface of XPS while the bottom line is the temperature on the external surface. The distance between adjacent lines (from the second line) is $1 \mathrm{~mm}$ (element size mentioned above). Experimental air and XPS surface temperatures at the inside of the box are used to calibrate the inside surface convection coefficient $h_{i}$. This calibration gave a $h_{i}=20 \mathrm{~W} /\left(\mathrm{m}^{2} \mathrm{~K}\right)$.

Fig.8 shows that the maximal internal surface temperature of the XPS changes from about $37.8{ }^{\circ} \mathrm{C}$ in Fig. 8 a to $40^{\circ} \mathrm{C}$ in Fig. 8 b. Due to differences in heat flow, the temperature drop over the wall is not doubled, as might be expected when the wall thickness is increased from $1 \mathrm{~cm}$ to $2 \mathrm{~cm}$. The actual temperature drop ratio is $16 / 12=1.3$.
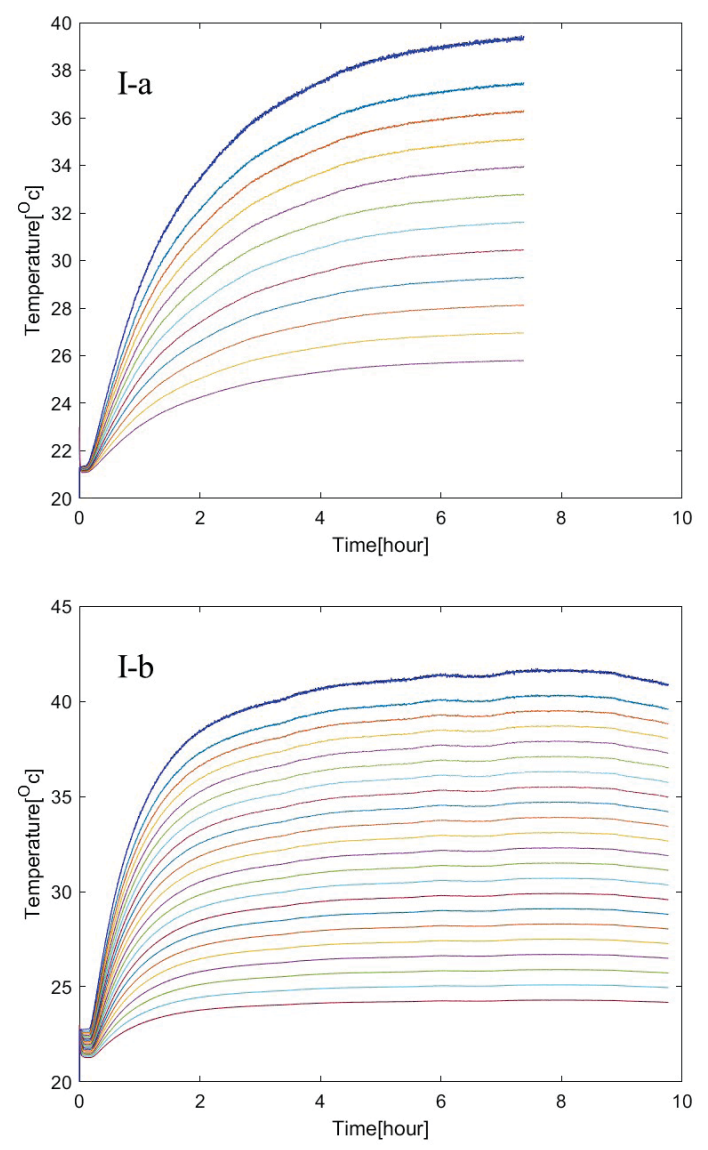

Fig. 8. Temperature profile of construction I from simulation;
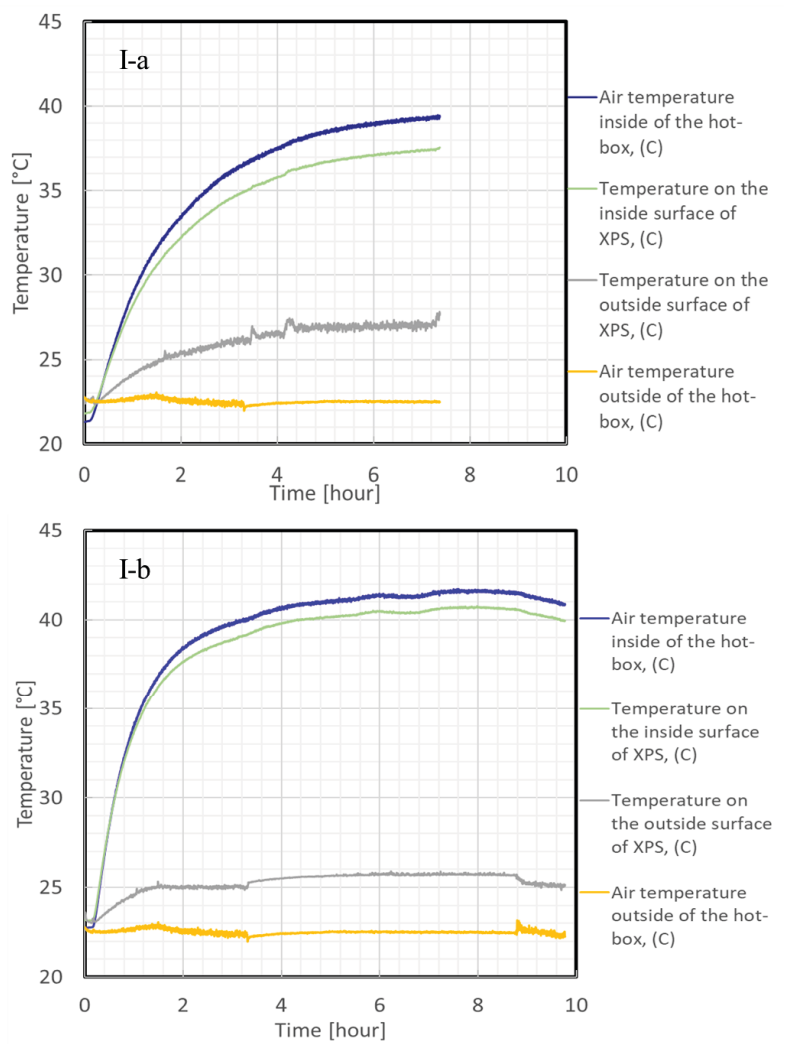

Fig. 9. Temperature profile of wall configuration I-a and I-b from experiment.

Experimental results for the cases I-a and I-b are shown in Fig. 9. For an XPS thickness increase from 1 $\mathrm{cm}$ to $2 \mathrm{~cm}$ the results are showing a similar increase as the simulations show, for the maximal inside XPS surface temperatures, $37.5^{\circ} \mathrm{C}$ and $40^{\circ} \mathrm{C}$, respectively. The temperature drop ratio is 1.5 , which is fairly close to the value 1.3 , obtained from the simulations.

Comparisons of Fig. 8 and Fig. 9, indicates that the simulation tool (eFEM) applied in this study can predict the experimental results well in the XPS cases.

\section{b) Two layers (Config. II - a and b)}

The temperature profiles from simulations for the two layers configuration II-a and II-b (See Fig. 5) are shown in Fig.10. The figures show a phase transition in Fig. $10 \mathrm{~b}$ for the configuration II-b (starting from about $26.5^{\circ} \mathrm{C}$ and ending at about $29^{\circ} \mathrm{C}$ ), but for the configuration of II-a in Fig.10a, there is no clear indication. The conclusion from this is that, the PCM layer should be put nearest to the temperature variation side in order to increase its heat exchange and storage ability. Therefore, only wall configuration II-b is performed experimentally. Fig.10 also shows that with the configuration of II-b, the system stabilizes faster. The XPS on the outside leads to a lower maximal 

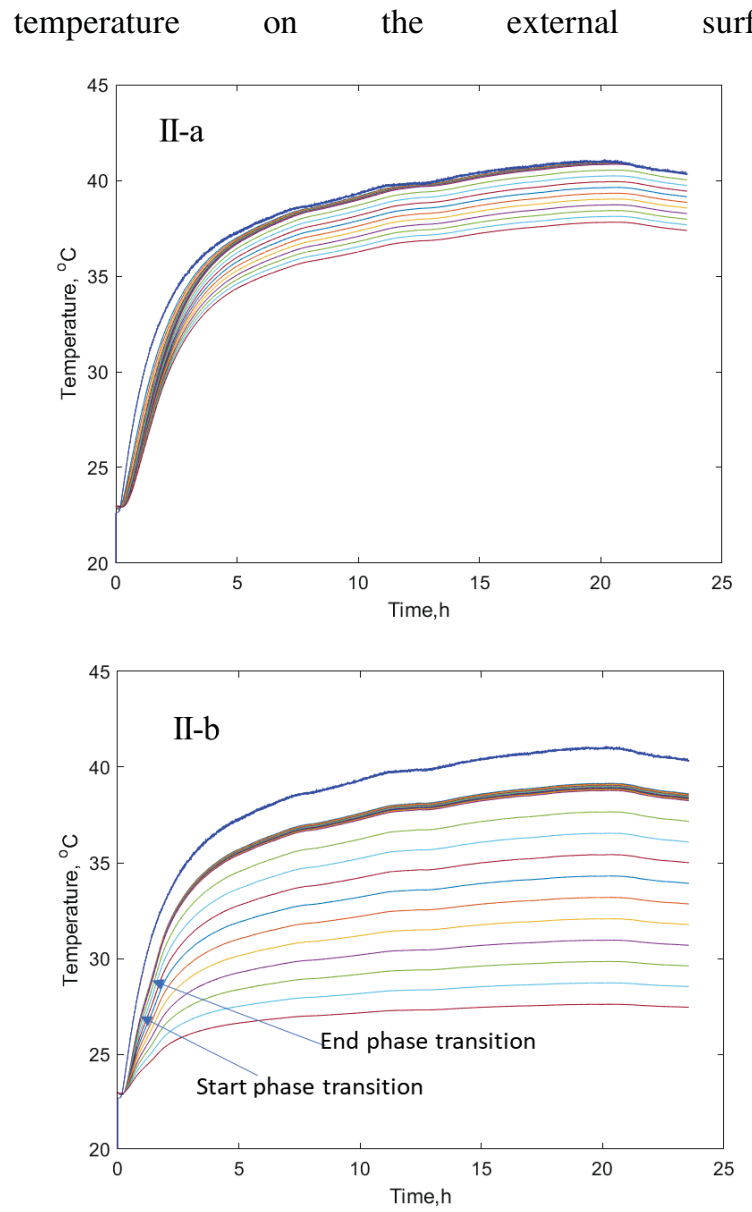

Fig. 10. Temperature profiles of wall configuration (II-a and IIb) from simulation.

The experimental results for wall configuration II-b are shown in Fig.11. The phase transition starts at about $27^{\circ} \mathrm{C}$ and ends at $32^{\circ} \mathrm{C}$. In comparison, the phase change in the simulation is not as distinct, which is a result of the influence of the $c_{p}$ curve shown in Fig.4. Therefore, in future studies, the specific heat capacity is suggested

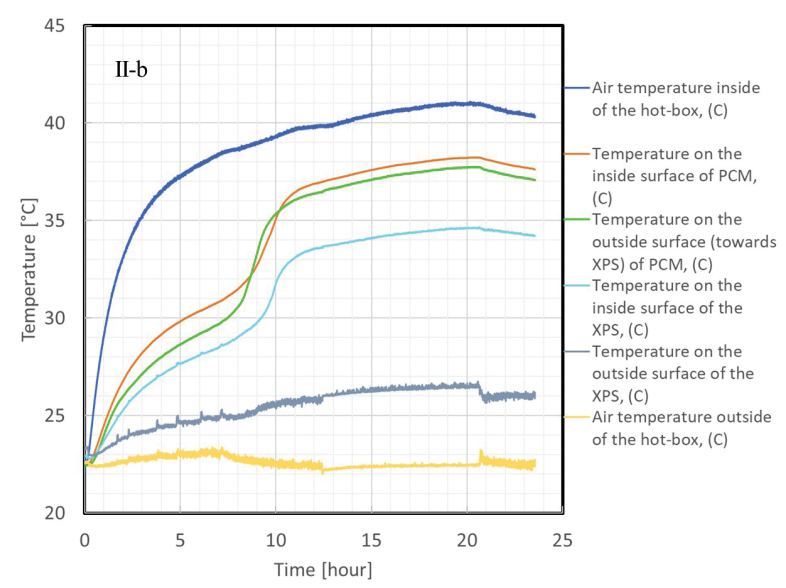

Fig. 11. Temperature profile of wall configuration II-b from experiment. to be measured, by differential scanning calorimetry (DSC). Improved knowledge of the transition performance may give a better agreement between the results of simulation and experiment.

\section{c) Three layers (Config. III - a, b and c)}

Fig.12 shows the simulated temperature profiles across the wall with the configurations of III -a, -b and -c (See Fig. 5). It contains two layers of XPS, one on each side of the PCM-layer. In Fig.12a, the thickness of the inner and outer XPS is $1 \mathrm{~cm}$ each, in Fig.12b, they are $2 \mathrm{~cm} / 1 \mathrm{~cm}$, and $1 \mathrm{~cm} / 2 \mathrm{~cm}$ in Fig. $12 \mathrm{c}$.

It appears from the comparison of Fig. $12 \mathrm{a}$ and $\mathrm{b}$ that with a thicker XPS layer the temperature difference over the wall increases. Going from configuration III-a to IIIc the outside temperature drops from $31^{\circ} \mathrm{C}$ to $30^{\circ} \mathrm{C}$. For the cases $b$ and $c$, where the thicknesses and material layers are the same, but with different sequencing, the temperature differences over the walls are about the same, $9^{\circ} \mathrm{C}$. Case III-c, with a thicker XPS on the outside, gives a lower temperature on the outside surface. By moving the $2 \mathrm{~cm}$ XPS from the outside, as in figure 12c, to the inside, as in $12 \mathrm{~b}$, results in a less distinct phase transition of the PCM. A thicker XPS on the inner side will also increase the time for a sudden temperature change to reach the PCM layer, which reduces the PCMs capacity to stabilize temperature fluctuations. In configuration a and c, where the inner XPS thicknesses both are $1 \mathrm{~cm}$, the starting point of the transition is more pronounced and happens earlier than in Fig. 12b.

Experimental results are only shown for configuration III-a, see Fig.13, where the end of the phase transition and the following temperature increase is observed at about $30^{\circ} \mathrm{C}$. The experimental results of configuration III-c show similar transition characteristics as III-a. However, for configuration III-b with a $2 \mathrm{~cm}$ XPS layer on the inside of the PCM, the temperature of the PCM only reached a maximal temperature of about $30^{\circ} \mathrm{C}$ and the succeeding characteristic temperature increase was therefore not observed. This shortcoming is due to the limitations of the power of the heating element inside of the Hot-Box. 

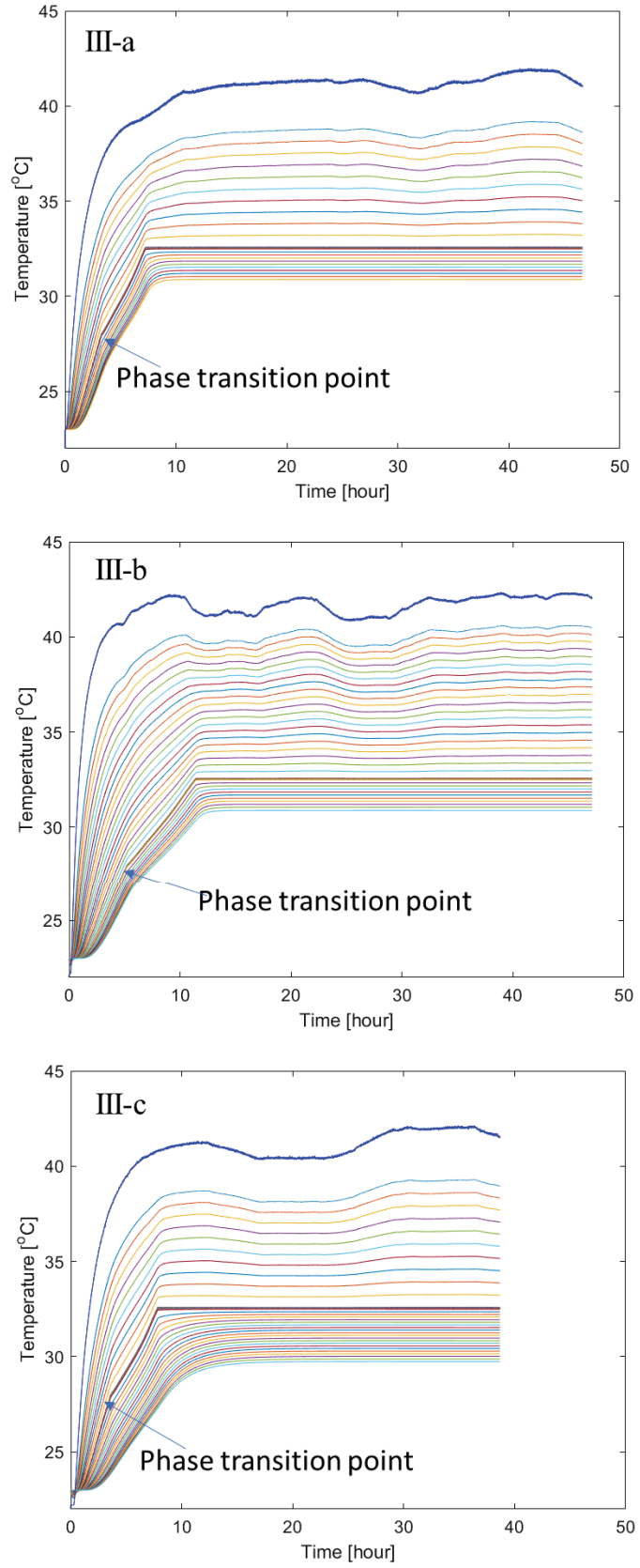

Fig.12. Temperature profile for the wall configuration III -a, -b and $-\mathrm{c}$ from simulation.

Fig. 13 also shows that the temperature difference over the outer XPS is comparable to the inner XPS layer, which is not the case for simulation results shown in Fig.12. That is because in simulations, the outside temperature of the hot-box is set constant as $23{ }^{\circ} \mathrm{C}$, however, in the experiment, the heating system keeps running until there is a final balance. It also shows from the comparison of the simulation and experimental results that, the phase transition starts later in experiment due to the limited control system.

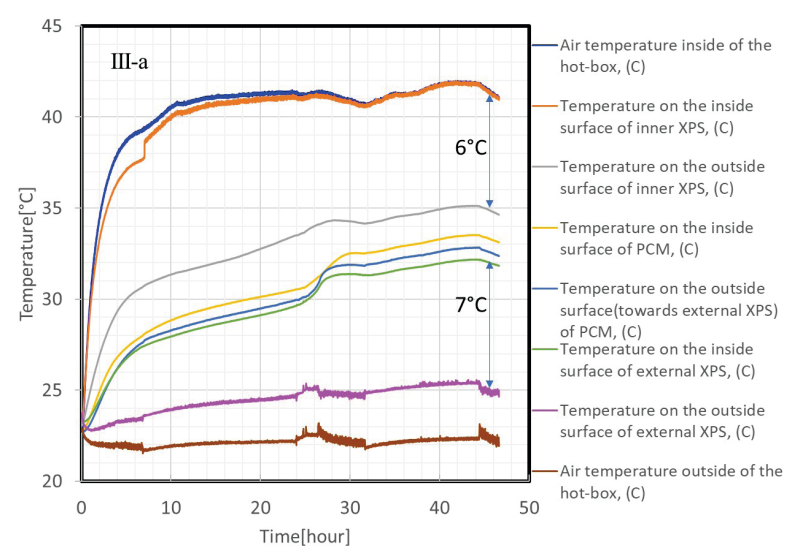

Fig. 13. Temperature profile for wall configuration III -a from experiment.

\subsection{Discussion}

We receive good conformity between the simulated and experimental data for the one layer case with XPS material. In the two layer case we still get acceptable temperature differences over the wall compared to the experimental, with a discrepancy within $1^{\circ} \mathrm{C}$. However, the PCM transition occurs much later in the experimental study. In the II-b case, the transition ends at about 1.5 hours and not until at 7.5 hours in the experimental study. The temperature discrepancy increases more in the three layer case, and also the time shift of the transition. In the experimental III-a case, it takes much longer time for the different layers in the wall to reach steady-state temperature conditions. One explanation of this is probably that we don't have perfect heat transfer between the layers inside of the wall, which is the case in the simulation. The absolute values of the specific heat capacity in the solid and the liquid phases, as well as the shape of the cp-curve, will have an impact of the simulations. We will therefore make measurements of the specific heat capacity.

In the coming development of the FEM model we will make a sensitivity study of the input parameters and variables and their significance on the results.

Both the convection on the inside and the outside walls are important for the overall heat transfer. A value of $8 \mathrm{~W} /\left(\mathrm{m}^{2} \mathrm{~K}\right)$ on the outside wall of the Hot-Box may be a somewhat low value in an office with a ventilation system running during parts of the day. Values of the convection coefficients have to be chosen with care in future and scaled-up cases for real buildings.

Even if our Hot-Box setup has its limitations, we emphasize that it is very informative and important to have experimental results to compare with. For future and more thorough work, it is desirable to have the possibility to control temperatures more accurate and measure heat flows experimentally. 


\section{Conclusions}

An explicit FEM model has been developed. The accuracy of this model has first been verified against the electrical circuit analogy model, and then validated against lab experiments where a small-scale hot-box was used.

Explicit FEM tools can be used to model/analyse the heat transfer performance of the wall layers but need to be further improved with sensitivity studies of the input parameters/variables and more accurate material properties;

By using the verified and validated FEM model a number of PCM wall configurations have been assessed. The study indicates that PCM material can be effectively used in walls as a layer for thermal storage and can in that way smoothen upcoming temperature variations;

The PCM layer should be put close to the temperature variation side in order to enhance the thermal performance.

In a future work, we will focus on the optimisation on the layer thicknesses.

The authors would like to thank the Northern Periphery and Arctic program, Kolarctic CBC Project: KO1089Green Arctic Building for financial support and the Sino-Nordic Research Center for Indoor Environment and Energy (SNRCIEE) for technical support.

\section{References}

1. S. M. Hasnain, Review on sustainable thermal energy storage technologies, Part I: heat storage materials and techniques, Energy conversion and management, 39 (11), 1127-1138, 1998.

2. S. M. Hasnain, Review on sustainable thermal energy storage technologies, Part II: cool thermal storage, Energy conversion and management, 39 (11), 1139--1153,1998.

3. L. Navarro, A. De Gracia, S. Colclough, et al. Thermal energy storage in building integrated thermal systems: A review. Part 1. active storage systems, Renewable Energy, 88, 526-547,2016.

4. L. Navarro, A. De Gracia, S. Colclough, et al. Thermal energy storage in building integrated thermal systems: A review. Part 2. Integration as passive system, Renewable Energy, 85, 1334$1356,2016$.

5. X. Wang, Y. Zhang, W. Xiao, et al. Review on thermal performance of phase change energy storage building envelope, Chinese Science Bulletin, 54(6), 920-928,2009.

6. J. Liu, Q. He, L. Yang, et al. Energy performance of a new Yaodong dwelling, in the Loess Plateau of China, Energy and buildings, 70: 159-166, 2014.

7. P. Uno, Concrete thermal mass and energy-efficient design, Constr. Review, 66(1): 159-166, 1993.

8. S. Goodhew and R. Griffiths, Sustainable earth walls to meet the building regulations, Energy and Buildings, 37(5): 451-459, 2005.
9. H. He, P. Zhao, Q. Yue, B. Gao, et al. A novel polynary fatty acid/sludge ceramsite composite phase change materials and its applications in building energy conservation. Renewable Energy, 2015, 76: 45-52.

10. Chandra S, Kumar R, Kaushik S, et al. Thermal performance of a non-air-conditioned building with PCM thermal storage. Energ Convers Manage, 1985, 25: 15-20

11. K. Ismail, J. Castro. PCM thermal insulation in buildings. International Journal of Energy Research, 1997, 21: 1281-1296

12. A. Athienitis, C. Liu, D. Hawes, et al. Investigation of the thermal performance of a passive solar testroom with wall latent heat storage. Building Environment, 1997, 32(5): 405-410

13. P. Marin, M. Saari, A. de Gracia, et al. Energy savings due to the use of pcm for relocatable lightweight buildings passive heating and cooling in different weather conditions. Energy and Buildings, 2016, 129: 274-283.

14. H. Yun, K. Ahn, S. Jang, et al. Thermal and mechanical behaviors of concrete with incorporation of strontium-based phase change material $(\mathrm{pcm})$. International journal of Concrete Structures and Materials , 2019, 13 (1): 18.

15. L. Cabeza, I. Martorell, L. Miro, A. Fernandez, et al. 1-Introduction to thermal energy storage (tes) systems, in: L. F. Cabeza (Ed.), Advances in Thermal Energy Storage Systems, Woodhead Publishing Series in Energy, 2015, pp. 1 -28.

16. V. D. Cao, S. Pilehvar, C. Salas-Bringas, et al. Thermal performance and numerical simulation of geopolymer concrete containing different types of thermoregulating materials for passive building applications. En. and Build-s, 2018, 173: 678-688.

17. X. Jin, M.A. Medina, X.S. Zhang, On the importance of the location of PCMs in building walls for enhanced thermal performance, Applied Energy 106 (2013):72-78.

18. A. Gounni, M.E. Alami, The optimal allocation of the PCM within a composite wall for surface temperature and heat flux reduction: An experimental approach, Applied Thermal Engineering 127(2017): 1488-1494.

19. N. Zhu, M.D. Wu, P.F. Hu, L.H. Xu, F. Lei, S.S. Li, Performance study on different location of double layers SSPCM wallboard in office building, Energy and Buildings 158 (2018): 23-31.

20. D. Heim and A. Wieprzkowicz, Positioning of an isothermal heat storage layer in a building wall exposed to the external environment, Journal of Building Performance Simulation 9 (2016):542-554

21. P. Gori, C. Gusyysti, L. Evangelisti, F. Asdrubali, Design criteria for improving insulation effectiveness of multilayer wall, International Journal of Heat and Mass Transfer 103(2016): 349359 . 